\title{
Characteristics of COVID-2019 in areas epidemic from imported cases
}

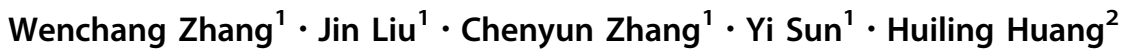

Received: 25 February 2020 / Revised: 6 July 2020 / Accepted: 9 July 2020 / Published online: 21 July 2020

(c) Swiss School of Public Health (SSPH+) 2020

\begin{abstract}
Objectives The outbreak of the 2019 novel coronaviruses disease (COVID-2019) in areas with epidemics due to imported cases is a cause of concern in China; however, few studies have reported on the prevalence of COVID-19 in these areas. Methods The number of diagnosed cases in Fujian Province was collected, and the time distribution of these cases was analyzed.

Results The results showed that the COVID-19 prevalence in areas with epidemics due to imported cases could be divided into two stages. The first stage was an outbreak dominated by imported cases, with the data showing an obviously skewed distribution. The second stage was dominated by nonimported cases with sporadic and low-level fluctuations. Moreover, the data demonstrated that the ratio of unexplained infections to nonimported cases was increasing.

Conclusions A two-stage outbreak in areas with epidemics due to imported cases, effective control of the "source of infection" and blocking of the transmission route can significantly minimize the peak height in the first stage and the spread of the epidemic in the second stage. Control of the epidemic in the second stage requires prevention and control of the aggregation of cases caused by unexplained infections.
\end{abstract}

Keywords 2019-nCoVs · Imported epidemic area · Outbreak · Case time distribution · Fujian Province · China

\section{Introduction}

Severe acute respiratory syndrome coronavirus 2 (SARS$\mathrm{CoV}-2$ ) was identified as the infectious agent causing an outbreak of viral pneumonia (coronavirus disease 2019, COVID-19) in Wuhan, China (Li et al. 2020; Special

Wenchang Zhang, Jin Liu and Chenyun Zhang authors have contributed equally to this work.

Wenchang Zhang

wenchang2008@126.com

Jin Liu

liujin@fjmu.edu.cn

Chenyun Zhang

zhangchenyun198724@126.com

Yi Sun

770240726@qq.com

Huiling Huang

huanghuiling1961@126.com

1 School of Public Health, Fujian Medical University, Xueyan Road No. 1, Minhou County, Fuzhou 350108, China

2 Fujian Medical University Union Hospital, Fuzhou 350001, China
Expert Group for Control of the Epidemic of Novel Coronavirus Pneumonia of the Chinese Preventive Medicine Association 2020). COVID-19, which is a new infectious disease, is highly contagious and spread quickly to other provinces in China and even to other countries (World Health Organization 2020). Fujian, a province on the southeast coast of China, is home to 38.56 million people. Since the outbreak of COVID-19 at the end of 2019, approximately $100,000-120,000$ travelers have entered Fujian from Wuhan. On January 22, 2020, the first imported case of COVID-19 was reported, and by February 21, 2020, a total of 293 COVID-19 cases had been confirmed in Fujian. Because the current knowledge of this virus is insufficient, research on the outbreak is very important but also very difficult. Moreover, this substantial risk to public health has attracted a great deal of attention from the research community. However, few studies exist on the prevalence of COVID-19 in areas with epidemics due to imported cases.

The corresponding author of this study is one of the members of the Special Expert Group (six persons in total), a group that is working for provincial governments to prevent and control COVID-19 in Fujian. The authors analyzed and evaluated the COVID-19 outbreak and its 
epidemiological trend in Fujian Province by performing an analysis of the temporal distribution of COVID-19 cases in the epidemic area, with the goals of revealing the characteristics of COVID-19 in an area with an epidemic originating from imported cases and providing a scientific basis for the formulation of prevention and control strategies for COVID-19.

\section{Methods}

The numbers of newly diagnosed and cumulative cases and epidemiological data of cases in Fujian Province were collected daily by officials (http://wjw.fujian.gov.cn/ztzl/ gzbufk/yqtb/) from January 22 to February 21, 2020. All the data were collected on the day of case identification, and the diagnosis was based on the diagnostic criteria for COVID-19 in China (including symptoms and nucleic acid test results) (http://www.gov.cn/xinwen/2020-02/20/con tent_5481071.htm). An imported case refers to a case in which a person has a history of travel from Wuhan; nonimported cases refer to local cases from December 2019, in which the individuals do not have a history of travel from Wuhan. Nonimported cases can be divided into those with a clear history of close contact with imported cases and those without a clear history of close contact with imported cases (referred to as unexplained infections). Based on these data and the analysis of the temporal distribution of COVID-19 cases, we investigated the spread and development of the COVID-19 epidemic in Fujian Province.

\section{Results}

\section{Temporal distribution of COVID-19 in Fujian Province}

As shown in Fig. 1a, the number of imported cases increased significantly from January 25, 2019, and maintained a relatively high level, even while fluctuating. The number of cases began to decline from February 4, 2020, and the spread of the epidemic slowed, with no new cases on February 19. The distribution of daily cases had an obviously skewed distribution. At the same time, a sigmoid (S-shaped) tendency of the cumulative incidence curve was observed (Fig. 1b).

Subsequently, on the 12th day after the diagnosis of the first imported case (February 3, 2020), an overlap of the two incidence curves appeared, indicating that the COVID19 outbreak had entered a nonimported stage (Fig. 1c). Up to this point, the outbreak could be divided into two stages: the first stage was dominated by imported cases and lasted from January 22 to February 3, 2020, during which a total of 194 cases were confirmed, and the second stage of the outbreak was dominated by nonimported cases and began on February 4, 2020, during which a total of 99 cases have been confirmed so far. The outbreak has entered another advanced stage.

\section{Analysis of the temporal distribution of imported COVID-19 cases}

To obtain a clearer picture of the trajectory of the outbreak, we observed the temporal distribution of imported cases. The results are shown in Fig. 2a. The initial imported cases were mainly detected between January 22 and February 4 and occurred on days 1-14 (163 cases in total). The data also showed that the peak ranged from 4 to 6 days (for example, from January 25 to January 27, 2020, 62 cases in total), and imported cases in this period accounted for $38 \%$ $(62 / 163)$ of the total cases in the first stage. However, the longest period was more than 20 days (February 10, 2020). The distribution of imported cases was essentially consistent with the incubation period of COVID-19 (Backer et al. 2020; Guan et al. 2020). The distribution of imported cases also had an obviously skewed distribution, and a sigmoid tendency was observed in the cumulative incidence curve.

\section{Analysis of the temporal distribution of nonimported COVID-19 cases}

The first nonimported COVID-19 case occurred on the fourth day after the report of the first imported case (January 26, 2020), after which the number of nonimported cases gradually increased (Fig. 3a). Thus far, the nonimported cases have exhibited a sporadic pattern without a peak. The number of new daily confirmed cases was higher 5-14 days after the first report of nonimported cases.

We also studied the temporal distribution of different exposure histories in nonimported cases. Since February 6, 2020, the number of unexplained infections has increased (Fig. 3b); in particular, the ratio of unexplained infections to nonimported cases has shown an obvious increasing trend (Fig. 3c).

\section{Discussion}

The epidemiological data on COVID-19 infections in Fujian Province were collected. The results indicated that the first imported case was detected on January 22, 2020, and the first nonimported case was detected on January 26, 2020, which was only 4 days later. According to the investigation, we also found that there was no direct epidemiological link between the first nonimported case and the first imported case. In fact, the first nonimported case 
Fig. 1 Time distribution of COVID-2019 from January 22 to February 21, 2020 in Fujian Province, China. a The daily new confirmed cases in Fujian Province; $\mathbf{b}$ The cumulative confirmed cases in Fujian Province; c The daily new imported/nonimported confirmed cases in Fujian Province
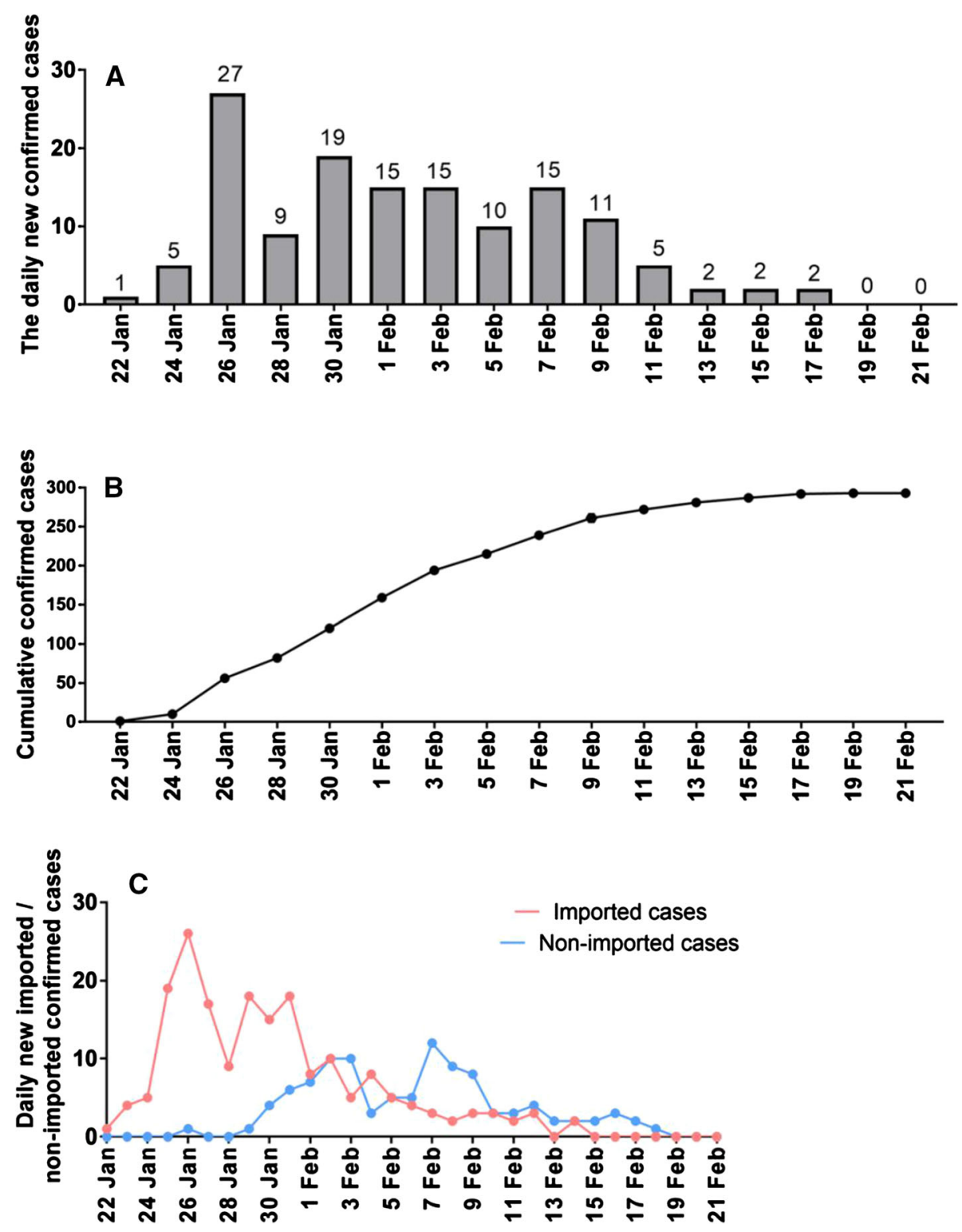

had had close contact with other confirmed imported cases of COVID-19, suggesting that while imported cases were occurring, the risk of local transmission already existed. By February 21, 2020, a total of 293 confirmed cases of COVID-19 (including 14 cases of severe or critical illness and 1 death) had been reported in Fujian Province. On January 23, 2019, major travel restrictions were introduced, limiting travel from Wuhan, and at the same time, Fujian Province began to implement the centralized investigation and registration of travelers from Wuhan. This indicated that when strict control over the imported cases was implemented as early as possible, the outbreak was effectively controlled for at least 1 month. Furthermore, since February 3, 2020, the daily number of nonimported cases has exceeded the number of imported cases (Fig. 1), suggesting that the epidemic in Fujian Province became dominated by nonimported cases. The data also showed the beginning of the epidemic stage of local transmission. Based on these findings, we determined that the COVID-19 outbreak can be divided into two stages: the first stage was dominated by imported cases, and the second stage is dominated by local cases. According to different stages of epidemic development, it is very important to public health to formulate and implement different prevention and control strategies.

Overall, the outbreak in this area, which originated from imported cases, has involved a transition from imported to nonimported cases. However, investigation of the temporal distribution of imported cases and nonimported cases led to more interesting observations. Imported COVID-19 cases dominated the outbreak in this area during the first stage. These imported cases mainly included people who were 
Fig. 2 Time distribution of imported COVID-2019 cases from January 22 to February 21, 2020 in Fujian Province, China. a The daily new confirmed of imported cases in Fujian Province; $\mathbf{b}$ The cumulative confirmed of imported cases in Fujian Province
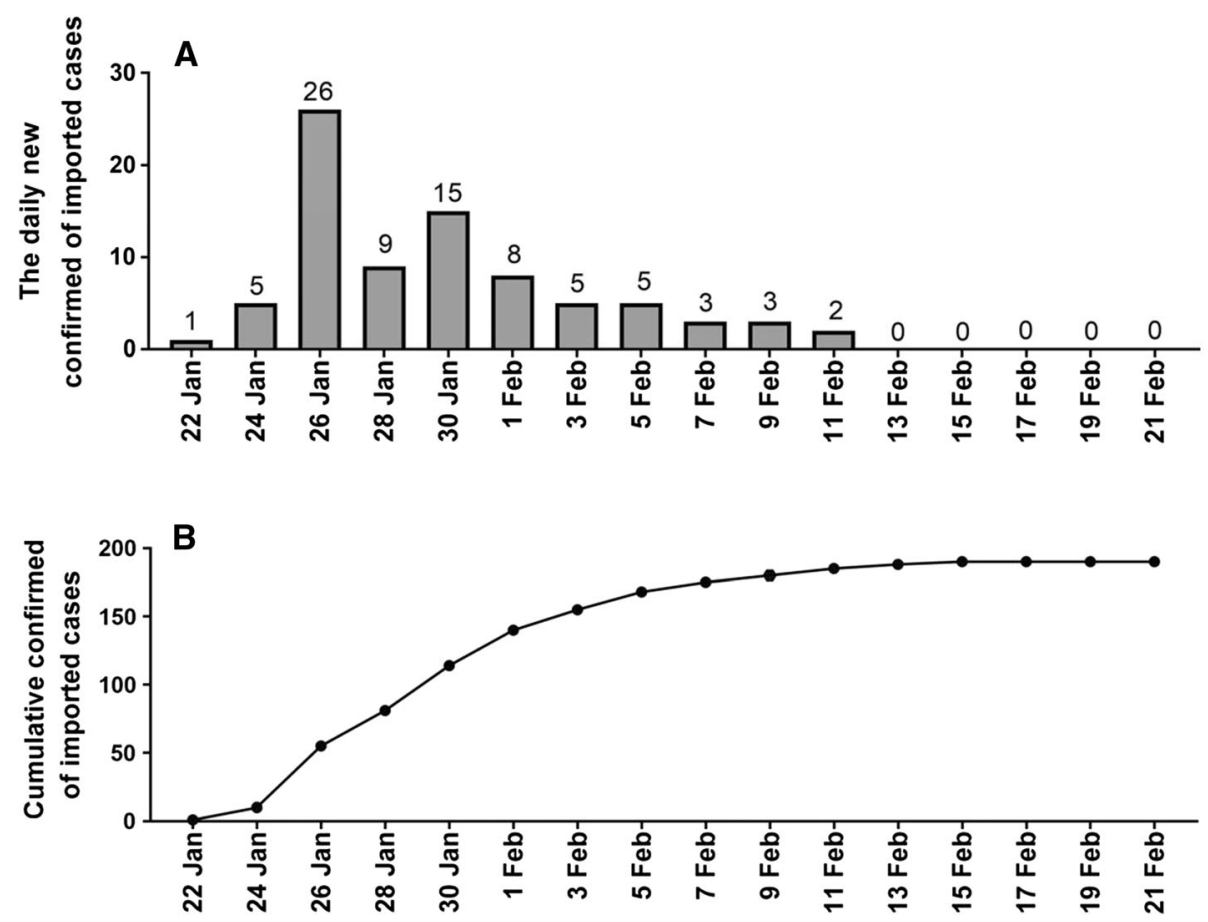

infected in Wuhan and then traveled to other places. The data from these cases showed an obvious skewed distribution, and a sigmoid tendency was noted in the cumulative incidence curve (Fig. 2). Specifically, the imported cases were mainly detected between January 22 and February 4, 2020 (163 cases in total), and the peak period ranged from 4 to 6 days after the onset, which then changed to a more sporadic pattern after 14 days. This distribution of imported cases was consistent with the incubation period of COVID-19. The COVID-19 incubation period is 1-14 days, and the mean incubation period is 4-6 days. Thus, observation of the pattern of data from January 22 to 28 enables the prediction of the pattern of the outbreak in the next stage with regard to imported cases of COVID-19. Specifically, given the strict interventions (such as cutting off transmission) and the observed distribution, we predicted on January 29, 2020, that the number of imported cases between January 29 and February 4, 2020, would not exceed the cumulative number of cases before January 29, 2020. Furthermore, the total number of imported cases was 81 from January 22 to 28 and 74 from January 29 to February 3. The above prediction of the number of cases provides an important basis for the advanced preparation of wards, beds and other medical facilities in the early stage.

Furthermore, the first nonimported case was detected 4 days after the first imported case (January 26), and the number of cases then gradually increased, leading to the next stage of the outbreak (February 3, after the two incidence curves overlapped). The new cases in this stage were occurring 5-14 days after the first nonimported case, which was consistent with the incubation period (7-14 days), without multiple peaks. If the reproduction number for COVID-19 was 2.68 (Wu et al. 2020), the total number of confirmed cases would have been close to ten thousand on January 29, 2020, in Fujian; however, the total number of confirmed cases was 101, and the number of nonimported cases was 87 , which is even lower than the number of imported cases (187) on February 1, 2020. Concerning the COVID-19 outbreak in Fujian, there were 293 cases at the time this article was written (February 21, 2020); thus, we believe that the management strategies implemented to control the source of infection and prevent transmission as rapidly as possible during the first stage were effective. In Fujian Province, which continues to manage the risk of imported cases, strict personal protection measures and limiting gatherings are the main strategies that are effective at controlling the spread of the epidemic. Although a few cases are detected each day, the temporal distribution of cases has become sporadic. We believe that the nonimported cases include unexplained infections; thus, the few cases currently observed may be unexplained infections. The data also showed that the ratio of unexplained infections to nonimported cases has been increasing, and more unexplained infections have been detected since February 6, 2020. Currently, there is no definite epidemiological exposure history for unexplained infections; they may be the result of contact with infected local individuals during the incubation period or exposure to missed cases (false negatives), which is considered to be another important source of SARS-CoV-2 infection. Therefore, control 
Fig. 3 Time distribution of nonimported COVID-2019 cases from January 22 to February 21, 2020 in Fujian Province, China. a The daily new confirmed of nonimported cases in Fujian Province; b The daily new confirmed of unexplained infections in Fujian Province; c The ratio of daily new confirmed of unexplained infections to daily new confirmed of nonimported cases in Fujian Province
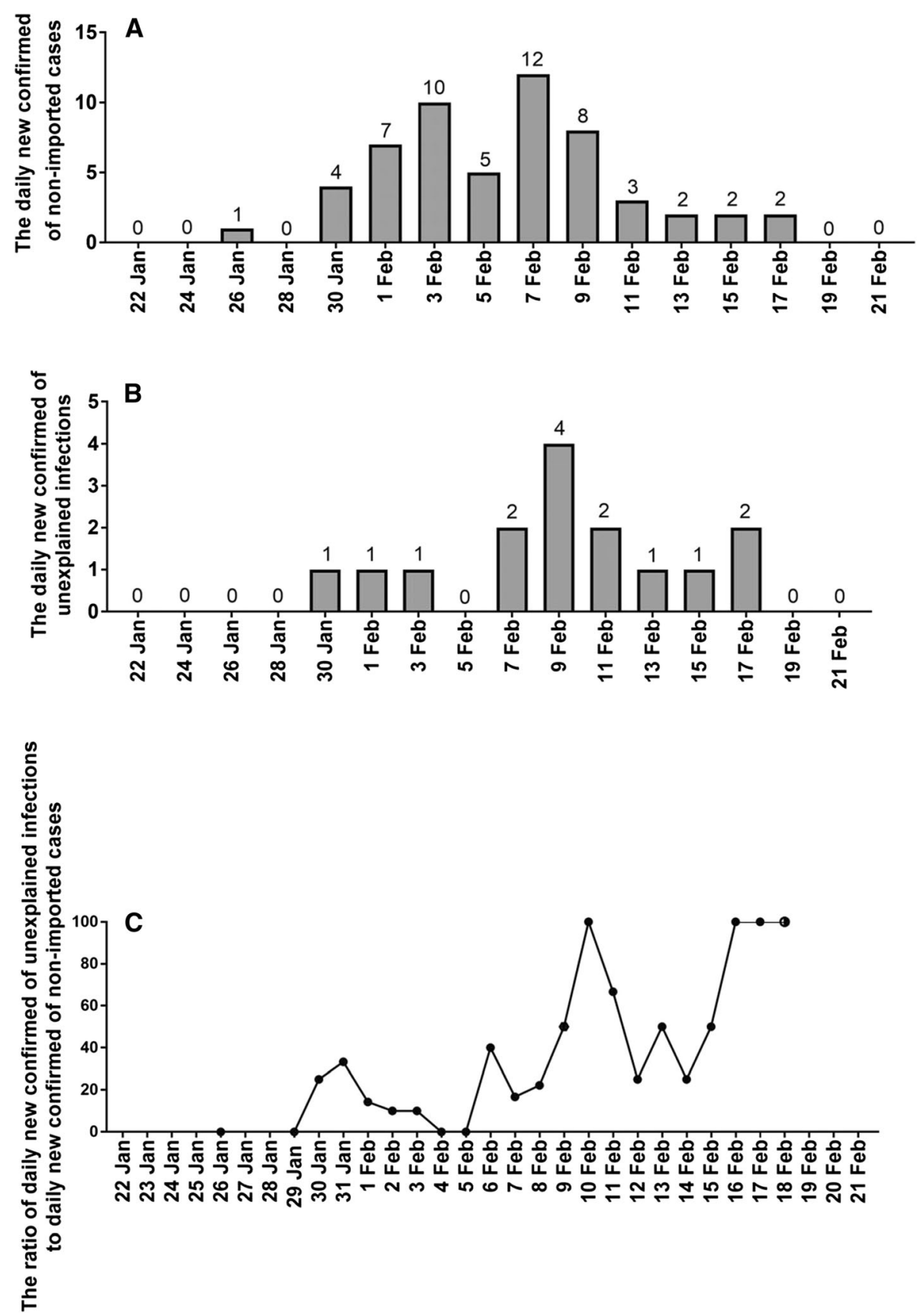

measures at the community level, including staying at home (with constant ventilation), attending fewer gatherings and implementing measures for personal protection, are the main effective strategies. Importantly, prevention and control of the epidemic at this stage require the reduction in unexplained infections.

In conclusion, the spread of COVID-19 in area with an epidemic originating from imported cases (such as Fujian) can be divided into two stages. In this setting, different prevention and control strategies should be implemented according to the characteristics of the different stages of the epidemic. Importantly, prevention and control of the epidemic in the ongoing second stage require the reduction in unexplained infections.

Acknowledgements We thank The Fujian Provincial Health Commission provided research data. We also thank the staff of the Fujian Provincial Center for Disease Control and Prevention for their hard work in the relevant epidemiological investigations.

Funding None. 


\section{Compliance with ethical standards}

Conflict of interest The authors declare no potential conflicts of interest.

\section{References}

Backer JA, Klinkenberg D, Wallinga J (2020) Incubation period of 2019 novel coronavirus (2019-nCoV) infections among travellers from Wuhan, China, 20-28 January 2020. Eurosurveillance 25:2000062. https://doi.org/10.2807/1560-7917.ES.2020. 25.5.2000062

Guan WJ, Ni ZY, Hu Y et al (2020) Clinical characteristics of 2019 novel coronavirus infection in China. $\mathrm{N}$ Engl $\mathrm{J}$ Med 382:1708-1720. https://doi.org/10.1056/NEJMoa2002032

Li Q, Guan X, Wu P et al (2020) Early transmission dynamics in Wuhan, China, of novel coronavirus-infected pneumonia.
N Engl J Med 382:1199-1207. https://doi.org/10.1056/ NEJMoa200131

Special Expert Group for Control of the Epidemic of Novel Coronavirus Pneumonia of the Chinese Preventive Medicine Association (2020) An update on the epidemiological characteristics of novel coronavirus pneumonia (COVID-19). Chin J Epidemiol 41:139-144. https://doi.org/10.3760/cma.j.issn.02546450.2020.02.002

World Health Organization (2020) Poverty. https://www.who.int/ docs/default-source/coronaviruse/situationreports/20200121sitrep-1-2019-ncov.pdf?sfvrsn=20a99c10_4. Accessed on 21 Jan 2020

Wu JT, Leung K, Leung GM (2020) Nowcasting and forecasting the potential domestic and international spread of the 2019-nCoV outbreak originating in Wuhan, China: a modelling study. Lancet 395:689-697. https://doi.org/10.1016/S0140-6736(20)30260-9

Publisher's Note Springer Nature remains neutral with regard to jurisdictional claims in published maps and institutional affiliations. 